\title{
Neuroendocrine Abnormalities in Drug Abusers and HIV-Infected Individuals: Cortisol Response to Cold Pressor Challenge
}

\author{
${ }^{1,2}$ Mahendra Kumar, ${ }^{1}$ Drenna Waldrop-Valverde, ${ }^{1}$ Adarsh M. Kumar \\ ${ }^{1}$ J. Benny Fernandez, ${ }^{1}$ Louis Gonzalez and ${ }^{1}$ Raymond L. Ownby \\ ${ }^{1}$ Department of Psychiatry and Behavioral Sciences \\ ${ }^{2}$ Department of Psychology University of Miami, School of Medicine, Miami, FL 33136
}

\begin{abstract}
Although endocrine abnormalities have been reported in HIV-1 infection, the role of risk factors is not understood. Injecting drug use (IDU) is an important risk factor for contracting HIVinfection and studies suggest that substance use may also be associated with endocrine dysfunction. In order to investigate hypothalamic pituitary adrenal (HPA) axis activity in this population, this study investigated cortisol response to the cold pressor challenge in IDUs with and without HIV infection. After controlling for the effects of gender, duration of marijuana use and time since the last use of heroin, the findings show that the pattern of cortisol response depended upon HIV serostatus. Cortisol levels peaked later in HIV+ IDUs and recovered at a slower rate than HIV negative IDUs. These findings support our hypothesis that dysregulation in HPA axis activity occurs in HIV infected IDUs.
\end{abstract}

Key words: Neuroendocrine abnormalities, drug abusers, HIV-infection, cortisol response

\section{INTRODUCTION}

A number of endocrine and metabolic abnormalities have been described among individuals infected with HIV-1 and these abnormalities may contribute to increased morbidity and mortality ${ }^{[1-4]}$. It has been proposed that HIV-1 infection may directly or in association with other factors bring about adverse effects on the neuroendocrine systems ${ }^{[5,6]}$ and in particular, on the hypothalamic pituitary adrenal (HPA) axis. Findings on the effects of HIV infection on cortisol, the most important regulator of HPA axis activity, have so far been inconsistent and have shown levels of cortisol to be increased ${ }^{[8-9]}$, normal ${ }^{[10]}$ or decreased ${ }^{[11]}$. However, cortisol response, rather than its level alone, reflects HPA activity, which consists of two distinct processes: (I) response of the hippocampushypothalamus-pituitary-adrenal axis to stressors resulting in increased secretion of cortisol and (2) the negative feedback inhibition of HPA activity by cortisol resulting in inhibition of continued secretion of cortisol.

Additionally, recent reports suggest that changes in HPA axis regulation may be a consequence of molecular mimicry in the HIV virus genome and the key control elements of the HPA axis ${ }^{[12,13]}$. For instance, viral proteins gag and Ltr regions may interact with the pro-opiomelanocortin (POMC) promoter normally regulated by corticotrophin releasing hormone $(\mathrm{CRH})^{[14,15]}$. Additionally, other viral auxiliary proteins, viral protein $\mathrm{R}, v p r$ and viral infectivity factor, vif, can interact with the glucocorticoid receptors ${ }^{[16,17]}$.
Moreover, earlier studies also reported that at least four pathologic mechanisms may cause many of the endocrinologic disturbances in HIV-infection and include: stress, activation of cytokines, tissue destruction by HIV associated malignancies and medications ${ }^{[6]}$.

There are multiple risk factors for contracting HIV infection and most of the earlier reports on endocrine effects have been conducted in individuals contracting infection via unsafe sex. One of the major risk factors for HIV infection is injecting drug use (IDU) ${ }^{[18,19]}$. Opiates, or heroin and specific forms of cocaine are the primary substances injected by IDUs although other forms of cocaine as well as marijuana are also used via other routes. All of these substances are likely to result in endocrine dysfunctions even in the absence of HIV infection. Cooper et al. ${ }^{[4]}$ reported that the adverse effects of opiates on cortisol levels may be related to opportunistic infections associated with drug abuse and in heroin abuse, and adrenal insufficiency may be a result of the effects of heroin on the neurotransmitters that regulate the secretion of corticotrophin-releasing hormones $(\mathrm{CRH})^{[20]}$. Cocaine abuse has also been related to abnormalities in HPA axis activity ${ }^{[21]}$.

Little is known about the cortisol response of HIV positive IDUs. Since opiates and other substances, as mentioned earlier, may also cause changes in HPA axis activity, it is not known how cortisol response to a stressor might be disrupted in individuals with both HIV infection and a history of injecting drug use. The present study assessed the cortisol response to cold

Corresponding Author: Mahendra Kumar, Department of Psychiatry and Behavioral Sciences, University of Miami, School of Medicine, 2029 Elliot Bldg. (M817), Miami, FL 33101 
pressor test, an $\alpha$-adrenergic challenge, in HIV positive and HIV negative IDUs. We hypothesized that the cortisol response to cold pressor would be dysregulated in HIV infected IDUs.

\section{MATERIAS AND METHODS}

Participants: Community residing men and women, who were enrolled as part of a larger study of endocrine responses to stress in persons with HIV-1 infection and a history of injecting drug use (IDU) were included in this study. For the present study, only HIV-1 positive and HIV-1 negative participants with a history of injecting drug use were included. Participants were within the age range of 18 to 50 and potential participants were excluded if they reported a history of head injury with loss of consciousness (> $30 \mathrm{~min}$ ), or a history of major psychiatric illness such as schizophrenia or bipolar disorder, hypertension or diabetes mellitus. Participants were considered injecting drug users if they had a history of regular injecting drug use defined as weekly drug injection for a period of at least 6 months. Additional use of other illicit substances via any route was not an exclusion criterion. Information on the duration and time since last use of heroin, cocaine/crack and marijuana was obtained from all participants. All participants were required to have abstained from drugs and/or alcohol for at least $12 \mathrm{hrs}$ prior to each session. This was verified by self-report and urine toxicology screens. If a participant was found to be acutely intoxicated, the appointment was rescheduled at a later date. HIV-positive participants were required to present evidence of their serostatus. Additionally, their plasma viral load was determined using PCR amplicor method (Roche Diagnostics; the Clinical Immunology Laboratory in the Department of Medicine, the University of Miami School of Medicine). Verification of HIV seronegative status was not available as part of this study. HIV-positive participants included in the study were free of any AIDS-defining clinical symptoms. This study was approved by the University of Miami Institutional Review Board. All participants gave informed consent prior to initiation of the study and were paid for their participation.

Cold pressor challenge: The cold pressor challenge was performed between 8:00 and 11:00 a.m. On arrival, an indwelling venous catheter for drawing blood was placed in the antecubital vein of each participant. After 20-min of rest in a reclined position, a 9-ml sample of blood was drawn in a tube containing EDTA to determine the resting, baseline level (T0) of cortisol. The cold pressor challenge was then administered.

Participants placed their entire hand in an ice-water mixture (3 parts ice and 1 part water) for 2 min.
Following completion of the cold pressor challenge, four additional blood samples were collected at $10 \mathrm{~min}$ (T1), $15 \mathrm{~min}$ (T2), $30 \mathrm{~min}$ (T3) and $50 \mathrm{~min}$ (T4) after baseline. Samples of blood were immediately centrifuged at $4^{\circ} \mathrm{C}$ and plasma obtained was aliquoted and stored at $-80^{\circ} \mathrm{C}$ until assayed.

Cortisol quantification: A solid-phase radioimmunoassay (RIA) was used to quantify plasma cortisol concentration using a commercially available kit (DSL, Webster, TX). Briefly, samples of plasma were defrosted, mixed and $25 \mu \mathrm{l}$ of plasma was added directly to cortisol antibody coated tubes, followed by the addition of $500 \mu \mathrm{l}$ of cortisol $\left[{ }^{125} \mathrm{I}\right]$ reagent and mixed. The mixture was incubated for $45 \mathrm{~min}$ in a water bath at $37^{\circ} \mathrm{C}$. The tubes were centrifuged and decanted and radioactivity was determined in an automatic gamma-counter (PerkinElmer, Model 1470). The minimum detection limit of this technique is $0.3 \mu \mathrm{g}$ of cortisol per $\mathrm{dL}$ of plasma. The intraassay and interassy coefficient of variance are $5.3 \%$ to $11.5 \%$.

Data analysis: Analyses were performed using the Statistical Package for the Social Sciences (SPSS) 12.0.1. Cortisol levels were quantified from plasma samples obtained at T0, T1, T2, T3 and T4 corresponding to baseline, $10 \mathrm{~min}, 15 \mathrm{~min}, 30 \mathrm{~min}$ and $50 \mathrm{~min}$ following the baseline sample.

Prior to evaluation of between group differences in cortisol response, the distribution of cortisol concentration at each time point was assessed. In all cases, evaluation indicated that values were not normally distributed. Values for cortisol concentrations were therefore natural $\log$ transformed; evaluation of distributions after transformation through inspection of plots of expected vs. actual values in a normal distribution confirmed the resulting distribution. Therefore $\log$ transformed cortisol levels were used in analyses.

To test our hypothesis that the cortisol response to stress would be affected by HIV serostatus, we used a repeated measures analysis of variance. We compared the cortisol response to cold pressor challenge between HIV positive and HIV negative participants. HIV serostatus was the between groups factor and time in min was the within groups factor. Bivariate analyses were performed to determine group differences in potential confounding variables. Spearman and Pearson correlations were used as appropriate to test for potential covariates for the repeated measures analysis. Variables tested were gender, age, race/ethnicity, years of education and duration and time since last use of marijuana, heroin and cocaine/crack. 


\section{RESULTS}

A total of 202 IDU participants completed the study: 109 (54\%) were HIV positive and 93 (46\%) were HIV negative. Demographic characteristics of the sample are presented in Table 1.

There was a significant difference in the racial/ethnic distribution of the HIV positive and HIV negative IDU groups. HIV positive participants used cocaine/crack significantly longer than HIV negative participants $(t=-2.950, \quad p=.004)$. HIV positive participants used cocaine/crack more recently than HIV negative participants $(t=-2.243, p=026)$ and HIV negative participants used heroin more recently than HIV positive participants $(t=3.788, p<.001)$. Gender, age and years of education did not differ between the groups.

Spearman and Pearson correlations to test for potential covariates indicated that gender, duration of marijuana use and time since last use of heroin were significantly correlated with cortisol levels. Repeated measures analysis of variance was thus used to evaluate group differences while controlling for these variables. Age, years of education, race/ethnicity, time since use of marijuana and cocaine/crack and duration of use of heroin and cocaine/crack were not significantly correlated with cortisol levels. Figure 1 illustrates the cortisol response curves of HIV positive and HIV negative participants.

Results of the repeated measures analysis of covariance revealed that cortisol levels changed significantly over time in both groups $(p<.001)$. The main effect for HIV infection status was not significant [F (1,170)=.008, p=.9300], however and more importantly, the pattern of cortisol change (HIV serostatus by time) differed substantially between HIV negative and HIV positive individuals $(p=.05)$. The average cortisol values for the HIV positive group increased more slowly and appeared to peak later than the average values for the HIV negative participants. Examination of these patterns using linear, quadratic and cubic orthogonal polynomials suggests that the differences resulted from rate of linear change over time as the quadratic and cubic terms were non-significant (Table 2).

Examination of the recovery phase of the curve (T2 to T4) shows that in HIV positive participants cortisol levels returned to baseline at a slower rate than those of HIV negative participants. HIV negative participants' values returned to baseline levels at T4. The HIV positive participants' cortisol levels did not return to baseline at the same rate. T4 cortisol levels of HIV positive individuals were significantly higher than their baseline (T0) cortisol levels (paired samples t-test; $t=-$ $3.174, p=.002)$. For HIV+ participants, cortisol response was not related to viral load (data not presented). These findings support our hypothesis that the pattern of cortisol response to stress is significantly related to HIV serostatus.

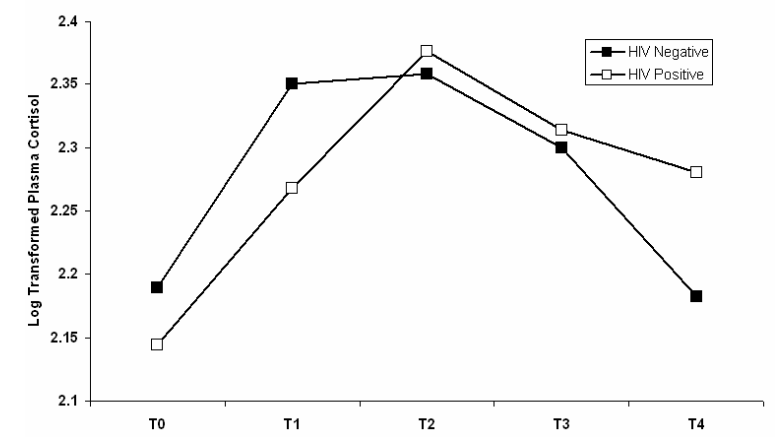

Fig. 1: Cortisol levels were assessed from plasma samples obtained at $\mathrm{T} 0, \mathrm{~T} 1, \mathrm{~T} 2, \mathrm{~T} 3$ and $\mathrm{T} 4$ corresponding to baseline, $10 \mathrm{~min}, 15 \mathrm{~min}, 30$ $\mathrm{min}$ and $50 \mathrm{~min}$ following the baseline sample, cortisol response to cold pressor challenge by HIV serostatus

Table 1: Demographic characteristics by HIV serostatus

\begin{tabular}{llll}
\hline & HIV Negative & HIV Positive & $p$ \\
\hline Mean (SD) Age & $39.08(6.96)$ & $39.72(5.85)$ & .478 \\
Mean (SD) Yrs Education & $11.82(2.01)$ & $11.74(2.10)$ & .074 \\
Gender & & & .907 \\
Male & 63 & 73 & \\
Female & 30 & 36 & \\
Race/Ethnicity & & & $<.000$ \\
Black & 37 & 83 & \\
White & 28 & 14 & \\
Hispanic & 28 & 12 & \\
\hline
\end{tabular}

Table 2: Repeated measures analysis: Cortisol change over time

\begin{tabular}{lccc}
\hline Effects & $d f$ & $\mathrm{~F}$ & $p$ \\
\hline Intercept & 1,170 & 1097.01 & $<.000$ \\
HIV serostatus & 1,170 & .008 & .930 \\
Gender & 1,170 & 4.811 & .030 \\
Marijuana duration & 1,170 & 5.279 & .023 \\
Heroin abstinence & 1,170 & 2.282 & .133 \\
Time (overall) & 4,167 & 11.139 & $<.000$ \\
Linear trend & 1,170 & 2.148 & .145 \\
Quadratic Trend & 1,170 & 37.998 & $<.000$ \\
Cubic Trend & 1,170 & 4.709 & .031 \\
HIV serostatus by time (overall) & 4,167 & 2.429 & .050 \\
HIV serostatus by linear trend ${ }^{a}$ & 1,170 & 6.622 & .011 \\
Marijuana duration by quadratic trend ${ }^{b}$ & 1,170 & 4.093 & .045 \\
Heroin abstinence by cubic trend ${ }^{c}$ & 1,170 & 5.288 & .023 \\
\hline$a$ & The tests for quadratic and cubic trends were not statistically \\
significant. & & & \\
$b \quad$ The tests for linear and cubic trends were not statistically \\
significant. \\
The tests for linear and quadratic trends were not statistically \\
significant.
\end{tabular}

\section{DISCUSSION}

The cold pressor test is the most commonly used stimulus to elicit hemodynamic, neurochemical and neuroendocrinologic responses. The present study carried out this test in order to investigate HPA activity among HIV-1 positive and HIV-1 negative IDUs using cortisol response to cold pressor challenge. The findings suggest that the cortisol response to the cold pressor test was significantly related to HIV serostatus. Findings also suggest that HIV positive IDUs take longer to return to baseline levels than HIV negative 
IDUs. These findings were found to be significant even after correction for gender, duration of marijuana use and time since last use of heroin. Some of the HIVpositive participants in this study were being intervened with anti-retroviral therapy and therefore their viral load may not represent true progression of HIV-disease. Plasma viral loads were not related to cortisol response. It may be concluded that the delayed cortisol response in HIV positive participants in the present study is a function of HIV infection rather than the viral load.

Cortisol response to cold pressor challenge has been used as a marker for HPA activity; however, there is a scarcity of data on the cortisol response to the cold pressor challenge in HIV positive IDUs. One of the earliest reports on cortisol response to the cold pressor challenge among homosexual HIV-1+ individuals was reported from this laboratory ${ }^{[7]}$ but the results were inconclusive since the challenge was carried out for only $15 \mathrm{~min}$. In the present study, cortisol response was found to be independent of race/ethnicity and years of education of the participants.

Although the mechanisms involved in the cortisol response to the cold pressor challenge remain uninvestigated, a number of possible mechanisms can be suggested. The cortisol response may be a function of increased tonic sympathoneural activity since release of cortisol from the adrenal cortex is under stimulatory modulation of sympathoneural innervation of the adrenal cortex ${ }^{[22]}$. The integration of the peripheral and central nervous system responses to cold pressor may also regulate the cortisol response. A careful examination of the cortisol response curve suggests that the rate of cortisol recovery was delayed among seropositive individuals. The recovery of cortisol level after a stressful challenge is a function of negative feedback inhibition of HPA activity ${ }^{[23]}$. In this process, cortisol inhibits the "stress" signals in the hippocampus, the hypothalamus and pituitary regulating the production of corticotrophin hormone to stimulate the adrenal cortex. The negative inhibition has been reported to be a function of cortisol binding to its receptors in the $\mathrm{CNS}^{[23]}$. Our unpublished preliminary data support this concept since we have observed that the glucocorticoid receptor mRNA is reduced in the hippocampal area of HIV-1+ individuals. Alternatively, the molecular homology among HPA regulatory proteins and HIV-genome may also play an important role. However, for this pathway to play an important role, it is necessary that these homologies are functionally active. It is also possible that these pathways may be independent of the negative inhibitory influence of glucocorticoids.

Although more studies are needed, our data support the concept that changes in the neuroendocrine system may be one of the major complications of HIV infection among drug abusers ${ }^{[4]}$.

\section{ACKNOWLEDGEMENT}

These investigations were supported by NIH grants RO1 DA 12792 and DA 13550. The authors are thankful to Mayuli Crespo, Peggy Gonzalez and Alison Mack for their assistance in data collection.

\section{REFERENCES}

1. Dobs, A.S., M.A. Dempsey, O.W. Ladenson and B.F. Polk, 1988. Endocrine disorders in men infected with human immunodeficiency virus. Am. J. Med., 84: 611-616.

2. Wahlstrom, J.T. and A.S. Dobs, 2000. Acute long term effects of AIDS and injection drug use on gonadal function. J. Acquir. Immune. Defic. Syndr., 25: S27-36.

3. Ferreiro, J. and H.P. Vinters, 1988. Pathology of the pituitary gland in patients with the acquired immune deficiency syndrome (AIDS). Pathology, 20: 211-5.

4. Cooper, O.D., T.T. Brown and A.S. Dobs, 2003. Opiate drug use: A potential contributor to the endocrine and metabolic complications in human immunodeficiency disease. CID, 37: 132-6.

5. Grinspoon, S.K. and J.P. Bilezikian, 1992. HIV disease and the endocrine system. N. Engl. J. Med., 327: 1360-5.

6. Grinspoon, S., 1996. AIDS: Endocrinologic and metabolic considerations. Ann. Med., 26: 321-323.

7. Kumar, M., A.M. Kumar, R. Morgan, J. Szapocznik and C. Eisdorfer, 1993. Abnormal pituitary-adrenocortical response in early HIV-1 infection. J. Acquir. Immune. Defic. Syndr., 6: 615.

8. Christeff, N., C. Michon, G. Goertz, J. Hassid, P.M. Girard, J.P. Coulaud and E.A. Nunez, 1988. Abnormal free fatty acid and cortisol concentrations in the serum of AIDS patients. Eur. J. Cancer Clin. Oncol., 24: 179-83.

9. Corley, P.A., 1995. HIV and cortisol connection: A feasible concept of the process of AIDS. Med. Hypothesis, 44: 483-89.

10. Kertzner, R.M., R. Goetz, G. Todak, T. Cooper, S.H. Linn, M.N. Reddy, H. Novacenko, J.B. Williams, A.A. Eharhardt and J.M. Gorman, 1993. Cortisol levels, immune status and mood in homosexual men with and without HIV infection. Am. J. Psych., 10: 1674-78.

11. Abbott, M., S.H. Khoo, M.R. Hammer and E.G. Wilkins, 1995. Prevelence of cortisol deficiency in late HIV disease. J. Infec. Dis., 31: 1-14.

12. Kumar, M., A.M. Kumar, D. Waldrop, M.H. Antoni and C. Eisdorfer, 2003. HIV-1 infection and its impact on the HPA axis, cytokines and cognition. Stress, 6: 167-72. 
13. Kumar, M., A.M. Kumar, D. Waldrop, M.H. Antoni, N. Schneiderman and C. Eisdorfer, 2002. The HPA axis in HIV-1 infection. J. Acquir. Immune. Defic. Syndr., 31: S89-93.

14. Licinio, J., P.W. Gold and M.L. Wong, 1995. A molecular mechanism for stress induced alterations in susceptibility to disease. Lancet, 346: 104-6.

15. Jin, W.D., A.L. Boutillier, M.J. Glucksman, S.R. Salton, J.P. Loeffler and J.L. Roberts, 1994. Characterization of a corticotrophin releasing hormone-response element in the rat POMC gene promoter and molecular cloning of its binding protein. Mol. Endocrinol., 189: 1377-88.

16. Kino, T., A. Gragerov, J.B. Kopp, R.H. Stauber, G.N. Pavalkis and G.P. Chrousos, 1999. The HIV1 viron associated protein $\mathrm{vpr}$ is a cofactor of human glucocorticoid receptor. J. Exp. Med., 189: 51-62.

17. Soudeyns, H., R. Geleziunas, G. Shymala, J. Hiscott and M.A. Wainberg, 1993. Identification of a novel glucocorticoid response element within the genome of human immunodeficiency virus type 1 . Virology, 194: 758-68.

18. Alcabes, P. and G. Friedland, 1995. Injecting drug use and human immunodeficiency virus infection. Clin. Inf. Dis., 20: 1467-79.
19. Sullivan, L.E. and D.A. Fiellin, 2004. Hepatitis C and HIV infections: implications for clinical care in injection drug users. Am. J. Addict., 13: 1-20.

20. Swerdlew, N.R. and G.F. Koob, 1985. Separate neural substrates of the locomoter-activity properties of amphetamine, heroin, caffeine and corticotrophin releasing factor in the rat. Pharmacol. Biochem. Behav., 23: 303-7.

21. Elman, I., H.C. Breiter, R.L. Gollub, S. Krause, H.L. Kantor, W.A. Baumgartner, D.R. Gastfriend and B.R. Rosen, 1999. Depressive symptomatology and coacine-induced pituitary-adrenal axis activation in individuals with cocaine dependence. Drug Alcohol Depend., 56: 39-25.

22. Bornstein, S.R. and G.P. Chrousos, 1999. Adrenocorticotropin and non-ACTH mediated regulation of the adrenal cortex: Neural and immune inputs. J. Clin. Endocrin. Metabol., 84: 1729-36.

23. Sapolsky, R.M., L.M. Romero and A.U. Munck, 2000. How do glucocorticoids influence stress response? Integrating permissive, suppressive, stimulatory and preparative actions. Endoc. Rev., 21: 55-89. 